\title{
An Exploration of How Artists Use Immersive Technologies to Promote Inclusivity, Diversity and Deep Public Engagement in Ethical Ways
}

\author{
Fion Gunn \\ Independent Artist \\ London, UK \\ fiongunn@live.co.uk
}

\author{
Maureen Kendal \\ Dreamstudio.io \\ London, UK \\ maureen@dreamstudio.io
}

\author{
Mehmet Mulla \\ Interface / Experience Designe \\ London, UK \\ mehmet.h.mulla@gmail.com
}

\begin{abstract}
The research investigates the relationship between artists, technology, and their audience. It documents their evolving creative practice as they engage with emerging technologies and where they use these as tools for access, exchange and deep engagement. The benefits and challenges of using immersive technology are explored. This includes user interface $I$ experience, interoperability and standardisation. Methods of collaboration across a range of cultures of usercommunities are explored. How do artists engage with some of these ethical questions as they use digital technologies and the capture of data? Our interviewees include artists, art professionals from galleries, museums and online virtual spaces. Interviewees were approached to include different technical, professional and social backgrounds.
\end{abstract}

Fine Art. Tilt Brush. Artificial intelligence. Virtual reality. Augmented reality. Ethics. User experience.

\section{BACKGROUND}

Everyday visual experience is conceived partly through our electronically mediated world, altering how we see and how we know. Computer Art was generated to adhere to a mathematical truth, registering sensory data beyond the scope of human vision. The artist pioneer Harold Cohen did not consider his work as Computer Art; rather he perceived all the diverse range of his work as his artist's practice. These artists, likewise, integrate emerging technologies within their practice thereby opening up new levels of engagement and reaching out to new audiences. Today this utilises emerging technical capabilities that use grid theory, 3D coordinates, edge detectors, neural networks enabling closed and open shapes; interactivity and rule-based systems. Since the 2000s, internet enabled and expert systems offer opportunities for artists to express their vision and engage with their audiences, including embedded sensor technology, interactivity and networks, neural networks, virtual headsets, augmented experiences that sew together the virtual and real physical experience.

Crucially artists can combine different technologies to create hybrid pipeline processes and unique systems to realise their innovative, aesthetic and valuable visualisations.

\section{METHODS I APPROACH}

The research team set out to explore evolving creative practices of artists using emerging technologies e.g. VR/AR/Al and other hybrid systems. Interviewees came from a range of backgrounds. Methods included physical and online semi- structured interviews, a quantifiable survey; researchers' participation at artists' events on immersive and related technologies, investigation of user experience of tool kits and pipeline process. An exploration of the benefits and challenges of immersive technology, user interface / experience, interoperability and standardisation.

\subsection{Artists}

Interviewees and survey respondents were artists and designers. Dynamic discussions across the research team and the interviewees sought to define the identity of being an artist and / or designer. 


\subsection{Questionnaire survey and semi-structured interview}

1. What was the trigger or inspiration that made you want to use immersive and emerging technologies - can you specify which you use? AR/VR/Al etc.

2. What have they brought to your work? Have they expanded your thematic or conceptual horizons?

3. What has been the impact on your material work, methods or practice? Do you integrate both tangible and digital ways of working and how?

4. Has your immersive work affected your audience and how?

5. What have been your main challenges using immersive technologies? And bearing these challenges in mind how you might work with software and hardware developers to improve these technologies for artists?

6. What are your future plans in this area?

\section{OBSERVATIONS AND INTERPRETATIONS}

Artists' responses encompassed the impact of emerging technology on society, ethical concerns around the use of personal data and data sets, representation, surveillance and the place of the 'human in the loop' within human-machine systems.

\subsection{Identity}

The term artist, much like 'designer' can have varying meanings. The definition lies in the liminal area between the creator's intentionality and the perceptions of the wider world. Many designers, who refer to themselves as User Interface or User Experience designers, have increasingly found themselves incorporating the other into their roles. Similarly, the further an artist expands their practice, incorporating different media and technology into it, the more the lines between artist and designer become blurred. At what point (if at all), does the use of immersive technology as part of an artwork, become the creation of an artist and not a designer and are the two synonymous when dealing with the use of the aforementioned technology?

\subsection{Perspectives}

Artist Nancy Baker Cahill has been working for ten years using VR and $A R$, she has delivered a TED talk on how she began using immersive technologies and why. "I started drawing in VR, because I wanted to generate an even more empathic connection in the literal physical body of the person who would stand in or move through my drawings" (Cahill 2018).

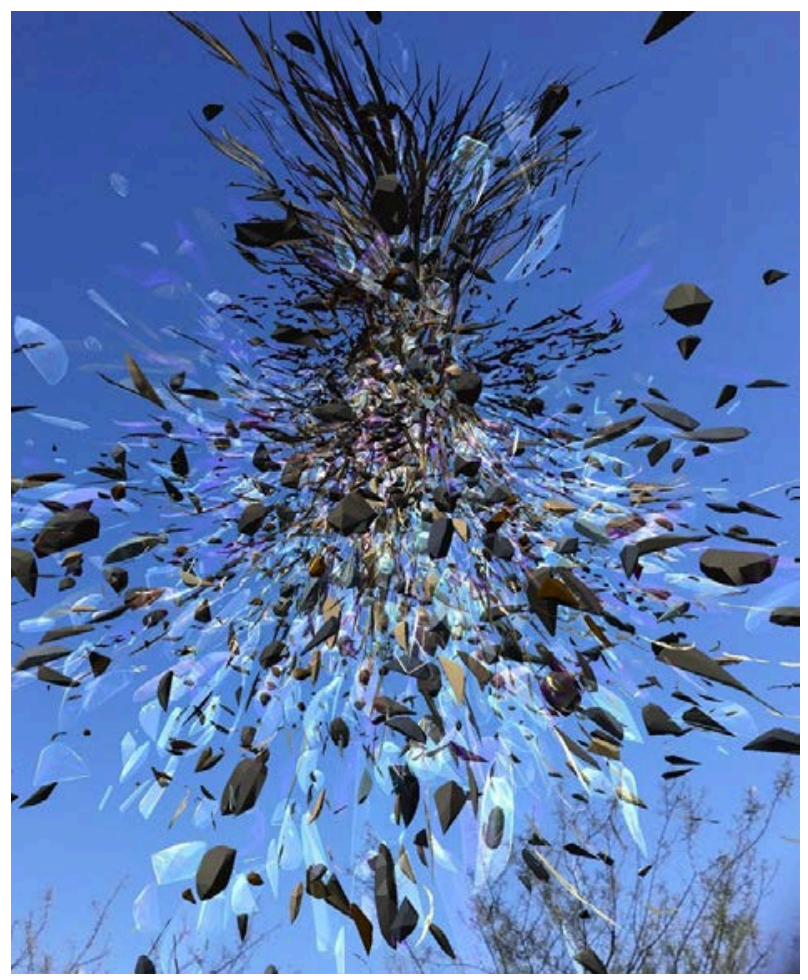

Figure 1: 'Legacy' is a site-specific work, which typifies Baker Cahill's approach.

When interviewed, both she and Sarah Rubidge, a choreographic installation artist, described their practices as "going back many years across a range of media ". Their shared inspiration involved a desire to expand horizons, to consider the emotional and sensory aspects of the resulting creations, the desire to 'move' audiences and engage with them more deeply. Baker Cahill describes the relationship between her material/tangible/analogue work and her digital work and says that "they are now inextricably intertwined". Rubidge had the desire "to share with audiences of dance performances the sensations and psychological state experienced by dancers when they are completely immersed in the act of dancing. This is often described by dancers and artists as being in the 'zone'". A sense of immersion as experienced by users of computer games and virtual immersive space is well documented by game theorists using Mihaly Csikszentmihalyi's experimental evaluation of Flow. The designer, Assa Ashuach commented, "I love to play with it Tilt Brush... the meaningful outcome... is playful experimentation in terms of $3 d$ sketching on human scale and to enable 3D immersive presentation." The VR artist Sean Rodrigo considers that this phenomenon of 'flow', immersion and 'being in the zone' is an approach for 'creating worlds for 
people'. In a real sense, this approach is synonymous with a spirit of welcome, the idea that artists can welcome viewers into the homes of their creations, the world of their ideas, their inner lives.

Several younger practitioners, whose background was devoid of physical making, found that exposure to immersive technology facilitated their engagement with material/tangible creation. This was a significant development for Ella Royer. Royer had not been allowed to make physical art at home as a child, because her mother had designated it as being 'too messy'. Royer now is confident to 'make stuff' and commented, "using VR/AR has helped me to return to the same materials which interested me before ... instead of making resin casts I use resin to make $3 D$ prints. The materials which interested me before, continue to interest me now".

In our survey (Q1) we wanted to find out if immersive technologies had cross-generational appeal for creatives.

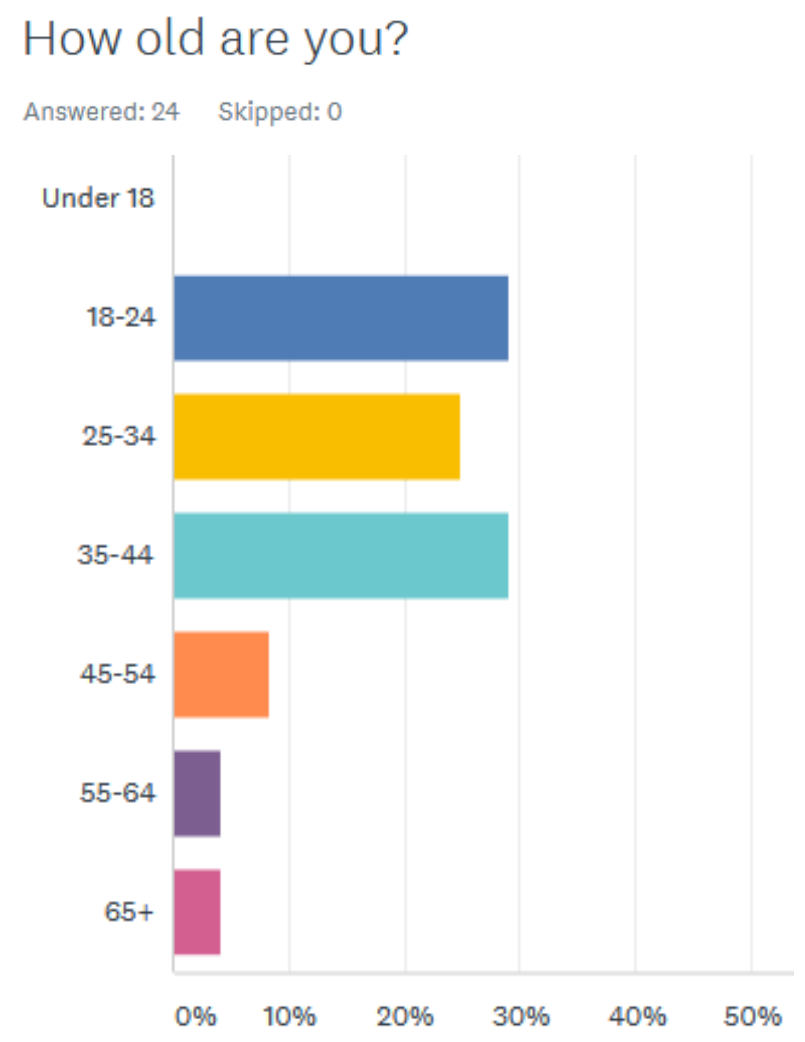

Figure 2: Survey results (Graph: age of survey participants).

The childhood environment was a significant factor with many interviewees. Sarah Selby described how "There were some key things, I was raised in a tech free household and had no access, I got access to everything simultaneously when I went to University at 19. I was questioning social interactions and how people interact with technology, so critical thinking was and is important for me". Julie Freeman, an artist who works with sound describes how she had "undiagnosed sight problems until the age of 10 and so sound was the primary sense through which I engaged with the world. I always use the term translating when I make work - I take data and then translate it into sound and visuals, sometimes directly sometimes indirectly. The work is an abstracted poetic treatment of the data and uses randomness". Many artists curate exhibitions, often in unconventional locations, artists/curators seek to create atmospheric environments, to create works which draw people in, and which include the narratives of others. It is the element of play inherent in the medium that facilitates open-ended creative activity for practitioners of all ages.

Immersive technologies provide a bridge to the evocation of childhood experience. From an early age, this ideal aesthetic and philosophical background these artists to interact with multilayered visual environments. These artists are influenced by complex and intricate artefacts from many cultures and periods of history.

VR \& AR, attracts a high proportion of women and older artists, who often do not have a tech or gaming background i.e. Rubidge. Sean Rodrigo reported that in his experience $40 \%$ participation of women in digital projects he ran, increased to $60 \%$ + when he introduced VR into his practice.

For Sarah Selby and Kadine James, the technology was not something they were 'expected' to get involved with, from both social and cultural perspectives it has fluid boundaries, it doesn't have to reside in galleries, museums or designated spaces and that's the attraction. An artist's role has and is often one of radical disrupter, subversive, agitator. The artist can offer an outsider perspective that transforms the habitual convention thus offering an alternative gestalt. The technology has for the most part, been designed by male coders and developers, the unconscious bias which this entails is increasingly obvious and increasingly problematic. Lucy Wheeler on the other hand came to use VR as a result of contact she had with artists at Tate Exchange where she had the opportunity to investigate the potential of imagined environments.

\subsection{Integrating the immersive}

The artist can engage with these emerging tools and capabilities through using high level design templates, pre-set tools or through manipulating the coding of algorithms, parameters, data sets or writing the code at a more granular level. The intention of the artist is to understand what it means to have consciousness, to respond and relate to self, the other and the environment. The artwork 
enables the audience to explore the inner world of the artist's engagement with human consciousness and engagement with this partially electronically mediated environment, including liminal subconscious processes and osmotic seepage between virtual and physical realities.

Each system has its own interface and thus its own experience. Although there are similarities, individuals, not familiar with one particular style, must face a learning curve for new methods of interaction. This does not include the variety of icons, and system graphics custom to each relative system. As designers, the ability to recognise and familiarize oneself with each new system typically becomes a habit as the need to use different software applications to complete different tasks becomes necessary.

Software development companies i.e. Adobe, are yet to transition into the immersive medium. However, their approach to creating interfaces for each different application with similar styles and interactions would be advantageous; this includes shortcut key binds. "Well applied conventions make it easier for users to go from site to site... there's a reassuring sense of familiarity" (Krug \& Black 2005). Alex Stanlake commented, "it was really "important to have universal icons and tools i.e. the 'undo' and 'redo' buttons. As a left-handed user I am very pleased with the interchangeability of the Oculus and Vive controls."

Artists will rarely go through this transition process as their form and process tends to be more physical, with the digital being limited to one particular application. Transitioning into a pipeline that includes interaction and production in extended reality can have a larger learning curve. Practical elements to which artists are accustomed allows them to learn in a way in which digital designers working in front of a computer screen do not. Using input devices such as keyboard and mouse as well as the addition of graphics tablets can have some advantages in the transition process.

What is striking with the interviewees is their perception of continuity of themes and concepts in their diverse practices. Most artists saw the expansiveness of immersive tech as a way to further their existing ideas and to pursue their longterm passions/obsessions. As Rubidge puts it "The thematics of my work have not really changed due to the technology. If they have changed, this is in response to world events, and social and cultural changes in society as a whole". Baker Cahill describes how the technology "has also indelibly altered and expanded the way I now draw on paper- I both conceive and represent space, mass, and velocity entirely differently". Striking too is a certain creative divergence, Wheeler and Jones striving for perfection and control whereas Selby, Freeman, Rubidge have an eye to the open-ended potential of immersion. This experimental approach is questioning and enables non-linear navigation and demonstrates the potential to reach new audiences.

\subsection{Sharing experiences}

It will always be a matter of perspective, how a technology benefits and or challenges us as its users. Ethical questions such as 'is it right for me to take a photo without permission', are more often than not dismissed in favour of a trigger finger approach. Both empathy and sympathy fail to inform any decision-making. Many go through the process of capturing footage and imagery of themselves but will dismiss/not consider the ideals of those they capture. The ramifications of this can cause a divide, between individuals, cultures and communities.

When interviewed, Andrew Carey, describes traditional Indian communities in New Mexico, "In the past, they let outsiders freely take pictures and those pictures were used by outsiders to label them as 'backwards' and 'pagan' and led to efforts to eliminate their customs and traditions". He later goes on to explain, "communities, such as the Navajo, have realized that outsiders regard their traditional clothing and appearance are seen as 'quaint'... They are poor communities with few resources, and they feel they have the right to some compensation if outsiders are coming into their community to gawk at them" (Carey 2020).

'Odyssey: Explorations', a collaborative project by artist Fion Gunn with Tate Exchange Liverpool in 2019 , encompassed in its first stage the creation of an interactive art exhibition, public and community workshops. Participants were introduced to AR and basic scanning techniques using 'Qlone' app. The second stage was the showcasing of a digital artwork created in VR with an accompanying AR art trail laid out across the Royal Albert Dock. The project attracted more roughly 3000 visitors, over 100 written comments as well as over 700 workshop participants.

Artists' experience has shaped their desire for a technology that they can work with. The public experience of immersive technologies as mitigated by artists was very positive. Whereas their experience of working with software/hardware developers was problematic. How are artists to address this dilemma? Building a genuine network of experienced creative artists, who have identified as such over a number of years could provide a way forward. These artists are not designers and have different requirements, so the process is slow and complicated. 


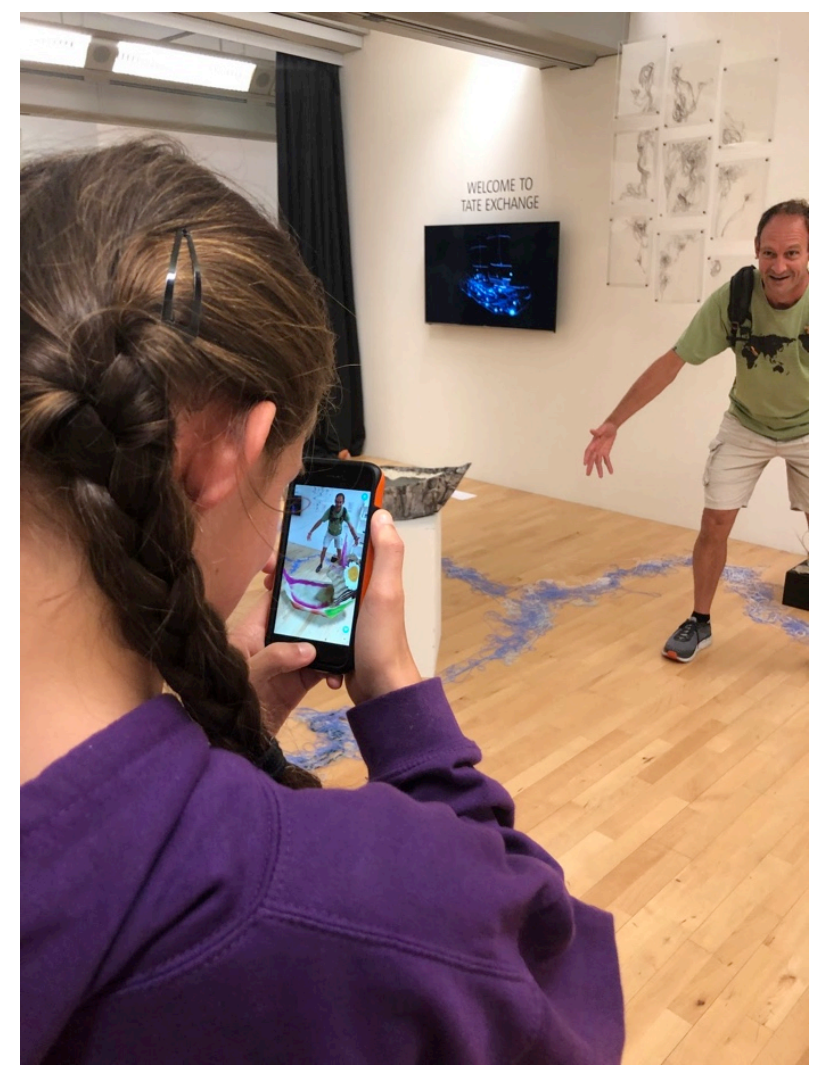

Figure 3: Young workshop participant using 'Qlone' to view scanned vessel she made at TEX as an AR pop up.

\subsection{Responses and challenges}

Interviewed artists described their experience of immersive technologies (positives and negatives) in ways, which echoed the experiences of Odyssey: Explorations project in Liverpool.

\section{Positives}

- "It was a magical experience to put on a headset for the first time - to see that other world opening up"

- "Tilt Brush software in particular is really accessible and it's possible to start creating straight away".

- Augmented Reality can add a rich, complementary and interactive dimension to exhibitions and take art out of galleries in an accessible way

- Being able to download simple AR apps, learn basic scanning techniques and pass that on to audiences and workshop participants is very empowering.

- It was reassuring that there were ways to find workaround solutions and artists are able to apply the same kind of lateral thinking that they use in other areas of their practice.

- It gave users confidence to start making things in other tangible/physical media
- Extending artists' skill sets have allowed those with audiences to extend their engagement with our work significantly.

- Artists are profoundly appreciative of the inclusive potential of immersive technology communities - the new groups and individuals encountered, of all backgrounds, genders and ages.

Negatives:

- As a majority of the artists in this paper mention - cost of the technology is a major factor.

- Difficulty using controls for anyone from a non-gaming background.

- Jargon is an issue, when artists encounter difficulties, they often don't even know what questions to ask in order to resolve them. They don't have the vocabulary to describe stuff, so they need to Google the most basic aspect of the problem and work from there, this was a widely experienced issue.

- Tech interface was another big factor, a specific problem with the Quest where the version of Tilt Brush it runs is not the same as Tilt Brush on tethered headsets. This has been very difficult for creating AR popups because models/sketches can't be uploaded as Objs.

- Working on a projects with developers can be a good way forward but there is often a disconnect between what the developer/coder is doing and what the user/artist is aiming for - cross disciplinary collaboration is tricky.

\subsection{Collaboration with developers}

Developers of these tools need to engage with conversations with their stakeholders. These include the users of the software tools such as artists, audience-participants who add usergenerated content and the curatorial eco-landscape of museums, galleries and cultural commentators.

Those with a curatorial perspective need to identify what makes these electronically mediated artworks, creatively and aesthetically valuable. Curatorial insights contextualise relationships between the artist's practice, emerging technologies, audience, and socio-political-economic realities. The artist's working practice requires a willingness to engage with a learning curve, and with the pipeline process, methods of working with a combination of technologies, a multidisciplinary skill-set, and collaborative practice with technology partners (V\&A 2020).

Ogi Damyan's comments were particularly apposite when he described the difficulties faced by users 
generally: "I found contributing to the codes, sending reports to engineers and engaging with people on social have been a really good way of mitigating these. The community is still relatively small and every voice and insight counts and helps.' Rodrigo points out that the technology is in its infancy and that "we should approach difficulties with a collaborative and appreciative spirit."

When asking the interviewees about how they would like to see software and hardware developers progress the technology a number of them: Selby, Wheeler, James felt that contributing codes would be useful. Wheeler was conscious of the slow learning curve for artists like herself, who started learning to code, 'it's a big investment of time' and all three felt that collaborations with startups are vital. Rubidge wanted to see artists working collaboratively with programmers to clean up bugs and that it was important "to find a realistic way of building in the 'cleaning' process into the artistic process." Stanlake flagged another issue standardisation. Whilst he did not necessarily want to see all interfaces and programmes standardised, as that might inhibit innovation, Technology needs to be more streamlined and flexible, to have more drawing tools within VR like Massless Pen and to draw/write rather than use a keyboard. Artists in general want a more open-ended approach, whereas technology developers are more solution driven, there is perhaps, an inevitable tension. Selby wished that developers would put aside their preconceptions and make their approach and process more accessible.

For artists like Ashuach, Elwes and Ridley, the future is working with a semi-autonomous methodology, a workflow of automated scripts with human input, allowing algorithms that grow. Ashuach acknowledges that immersive technology and Smart systems/ Al are two separate technologies. Currently there is a lack of interoperability standards across emerging technologies. Ashuach works with developers to invent new methods and tools. All the artists did agree on the requirement for developers to make tools and programmes more intuitive, with Royer remarking that they need 'to study the human body and movement and respond to it'. Jones was more demanding and was adamant that 'the tech shouldn't be a barrier, we shouldn't have to notice it. I want the tech to be there when I want it, how I want it.' On the other hand Rodrigo, a VR specialist urges patience, appreciation of the new technology and moving forward in a spirit of collaboration.

\subsection{Audience engagement}

These artists felt that their audiences have expanded, when they started using immersive technology. The potential to bring the experience of art out of galleries, museums and certain restricted social environments is important for all of us. It restores the primary relationship between artist and viewer, to one where the mitigating institutions are no longer given the power to step between them. In discussions with Rubidge, Baker Cahill, Wheeler and Ashuach, there was consensus on how the expansiveness of scale is made possible by the immersive and this has a significant impact on viewers.

Artist Julie Freeman's comment is a counterbalance to how the importance of audience interaction is often indiscriminately embraced, she describes how "there's a big pressure to make work interactive but then it can then be reduced to the level of a photo op and people are not really engaging with it in a meaningful way. I moved away from that. However, since I began to work in VR I have had to reframe the issue because it's not possible to make a VR work that's passive, the immersion is primary and it's important that the audience can navigate it in a seamless way."

There was consensus that, although VR was an amazingly useful creative tool, for widespread public engagement/audience interaction, AR, Al, animation and 3D scanning/printing were more significant and impactful, despite the occlusion problems with AR. Audience engagement also requires respectful consideration of their individual rights and requirements.

In our survey (Q6) we were able to establish a very strong consensus for the positive impact of immersive technologies on audience engagement. Immersive technology has enabled me to engage with audiences in a more effective way.

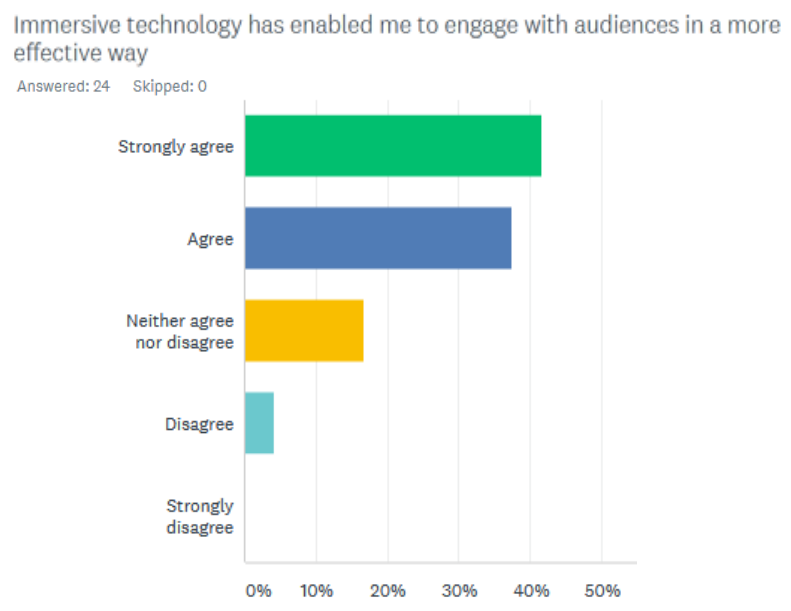

Figure 4: Survey results (Graph: survey participants engagement with their audience).

Whilst many audiences become accustomed to being captured, especially in more developed/populated areas, the right to choose is an important one, one's identity and in particular its capture as data in any type of media must be 
considered when developing any form of digital art. The legal and ethical rights of the audience who may be featured or be participants in immersive and extended realities need to be considered. Immersive environments provide the capacity for individuals to explore, work and play in. Ethical and legal issues open themselves to a new level of inquiry.

The concept of "Holoportation", an end-to-end system for Augmented and Virtual Reality telepresence, is currently being developed by Microsoft and others. Some companies offer 'free' access to the system being developed in exchange for use of the recorded image data of the user. This seemingly generous act might result in a potentially unethical use of the results. Necessary according to the law, data protection etc. If a specific user agreement stating what will happen with recordings and the fact that they are being recorded is not in place, then the potential for misuse is increased.

Furthermore, the recorded data would then be owned by the company, which could charge for its use. What are the implications for copyright? Does the company now own the digital 3D copy or just that specific recording? This might be problematic if a recording of the same person is made by two different companies. The individual needs to be protected from data misuse by the company, no matter what their intentions.

Elwes and Ridley are experimenting with combining electrically-mediated visualisation and smart system technologies - pointing us to the tension between control, predictability and entropy. Elwes called his latest work CUSP: "because it's on the cusp of two different states meeting in the technological and natural worlds. It brings them together. Cusp is also a mathematical function, so it has these different connotations...I think we are creating artificial intelligence to understand ourselves. There's a danger of anthropomorphising something that is just a mathematical process."

Anna Ridley's 'Laws of Ordered Form', FebruaryMarch 2020, explores image datasets. Ridley notes: "Materiality is one of the most contested concepts in contemporary art... has not to my knowledge been applied to work produced by neural nets." She sets out to explore "Can a GAN or indeed training set become a "wilful actor and agent within artistic processes' as other materials are? Whilst there is a certain amount of control afforded to the artist through the creation of a training set (images, labels), a GAN allows a quality 'where materials develop uncanny lives of their own and display their power to metamorphose, sometimes leading to images which might be labelled as ruined, decaying and decomposed in antithesis to the polish of 'consumed' images."' Ridley's work on 'misremembering and mistranslating' stands as a valuable testimony of this antithesis between the consumption of the perfect and ordered and an entropy towards unpredictability, anomalies and the random.

\section{CONCLUSION}

The divergence between the vision of fine artists and commercial artists hinged around concerns of open-endedness and perfection. Many of these fine artists focus on exploration, randomness and poetic envisaging. This artists' feedback could potentially shape how developers design the software. Our research has demonstrated that there is a need for interoperability, standards and platforms across emerging technologies.

Enablers would include:

1. To enable generative design to be demonstrated within 3D Virtual Reality experiences.

2. To code in and control measures of entropy, randomness, unpredictability into virtual reality tools

3. To code in hybridity between different categorisations of tools, patterns and presets

4. To re-write technical vocabulary for artists

5. New software versions to be compatible and not to lose previous functionality e.g. new untethered headsets

6. File formats to be standardised for interoperability

7. Lower entry costs and technical support to reach out to wider audiences

8. To build in ethical controls to protect individual data

The need for artist, designer:

1. To work collaboratively with developers and coders on different levels of code, from top end pre-sets and template tools, to using meta-code and then down to basic code.

2. To work with developers and coders, using playful experimental sandboxes, not just adhering to strict technical specifications or pre-set media conventions.

3. To combine the proliferation of design possibilities afforded by machine-learning with artists' intentionality.

4. To collaborate with developers to invent meta-languages to describe how computer programmes may 'think' when using neural nets. 


\subsection{The experience}

Artists use technology to further their practise, the method they take adheres to the pipeline available, a universal / consistent style for working with different tools, notably through the interface will provide them with an efficient means of producing their work whilst reducing the time needed to develop a new knowledge and approach to access and use new software.

Similarly, the ethics surrounding usage and permission can offer an appropriate level of comfort for those being captured if their considerations are taken into account, standards for capture could allow for more freedom in the artists process whilst restricting any unlawful attempts to create work at the expense of someone's privacy.

\section{REFERENCES}

Ashuach, A. (2020) interviewed by Maureen Kendal, 6th March 2020.

Cahill, N.B. (2018) Augmented Reality (AR) as an Artist's Tool for Equity and Access',

TEDxPasadena.

https://www.ted.com/talks/nancy baker cahill aug mented reality ar as an artist $\mathrm{s}$ tool for equity and access September 2018 (retrieved 15th March 2020).

Cahill, N.B. (2020) interviewed by Fion Gunn, 27 January 2020.

Carey, A. (2020) interviewed by Mehmet Mulla, 8 March 2020.

Damyan, O. (2020) interviewed by Fion Gunn, observed by Mehmet Mulla at Realities Centre London, 14 February 2020.

Elwes, J. (2019) in conversation with Maureen Kendal, November 2019, Edinburgh, Beyond Conference.

Freeman, J. (2020) interviewed by Maureen Kendal and Fion Gunn, 20 February 2020.

Fujita, G (2018) Quill Tutorial: Working in Quill 1.8. https://www.youtube.com/watch?v=1SMuD7yx8Hg $\underline{\mathrm{\&}=8 \mathrm{~s}}$ (retrieved 1st March 2020).
James, J. (2020) interviewed by Maureen Kendal and Fion Gunn, 14 February 2020.

Jones, R. (2020) interviewed by Fion Gunn, observed by Mehmet Mulla, at Realities Centre London, 13 February 2020.

Krug, S. and Black, R. (2005) Don't Make Me Think!: A Common Sense Approach to Web Usability, Indianapolis, IN: New Riders Publishing.

Maheshwari, M. (2019) Interview with Jake-Elwes on his CUSP exhibition 22 February 2019.

https://cdn.zabludowiczcollection.com/files/FINAL ZC Invites-Leaftlet Jake-Elwes Digital.pdf

Odyssey: Explorations (2019) Fion Gunn, Tate Exchange Liverpool, Tate and Open Eye Gallery Liverpool July/August 2019.

Orts, S. et el. (2016) Holoportation: Virtual 3D Teleportation in Real-time. doi: 10.1145/2984511.2984517

Ridler, A. (2020) http://annaridler.com/gans-in-art

Rodrigo, S. (2020) interviewed by Fion Gunn, 23 February 2020.

Royer, E. (2020) interviewed by Fion Gunn, observed by Mehmet Mulla at Realities Centre London, 13 February

Rubidge, S. (2020) interviewed by Fion Gunn, 11 February

Selby, S. (2020) interviewed by Maureen Kendal and Fion Gunn, 14 February 2020.

Stanlake, A. (2020) interviewed by Fion Gunn, observed by Mehmet Mulla at Realities Centre London, 13 February 2020.

V\&A (2020) Pre-histories and Futures of Machine Vision, 28th February. Funded by Vasari Research Centre, Birkbeck University. Speakers included digital scholars Zabet Patterson, Joel McKim, V\&A curators Douglas Dodds and Natalie Kane and contemporary artists Anna Ridler and Alan Warburton.

Wheeler, L. (2020) interviewed by Maureen Kendal and Fion Gunn, 14 February 2020. 